\title{
Heavy fermion quantum criticality: The party is just beginning
}

\author{
Yi-Feng Yang ${ }^{1,2,3 *}$ \\ ${ }^{1}$ Beijing National Laboratory for Condensed Matter Physics, Institute of Physics, Chinese Academy of Sciences, Beijing 100190, China; \\ ${ }^{2}$ University of Chinese Academy of Sciences, Beijing 100049, China; \\ ${ }^{3}$ Songshan Lake Materials Laboratory, Dongguan 523808, China
}

Received April 13, 2020; accepted April 21, 2020; published online May 15, 2020

Citation: Y.-F. Yang, Heavy fermion quantum criticality: The party is just beginning, Sci. China-Phys. Mech. Astron. 63, 117431 (2020), https://doi.org/10.1007/s11433-020-1567-7

Recently, Nature reported the discovery of a ferromagnetic quantum critical point (FM QCP) in the heavy fermion compound $\mathrm{CeRh}_{6} \mathrm{Ge}_{4}$ [1]. This is the first discovery of such kind in a stoichiometric compound. The FM order is suppressed at a critical pressure of $0.8 \mathrm{GPa}$, beyond which the ground state turns into a Fermi liquid. Previously, Science had also reported the observation of a FM QCP, albeit in the doped compound $\mathrm{YbNi}_{4}\left(\mathrm{P}_{0.92} \mathrm{As}_{0.08}\right)_{2}$ [2], where the As doping provides a negative chemical pressure. The current discovery in $\mathrm{CeRh}_{6} \mathrm{Ge}_{4}$ avoids the possible disorder effect and adds a clean and new member to the families of heavy fermion quantum criticality.

A QCP marks a continuous phase transition at zero temperature and can be achieved by tuning external control parameters $(\delta)$ such as the pressure, magnetic field, or doping. At finite temperatures, it induces a funnel-shaped region on the $(T, \delta)$ phase diagram, where low-energy quantum critical fluctuations can cause anomalous temperature dependence in thermodynamic and transport quantities. For example, in $\mathrm{CeRh}_{6} \mathrm{Ge}_{4}$, we have the resistivity $\rho \sim T$ and the specific heat $C / T \sim-\ln T$ in the critical region, which are different from the expectation of a Fermi liquid. This anomalous normal state is therefore called a non-Fermi liquid (NFL). Superconductivity often appears around the QCP.

Heavy fermion materials, owing to their rich competing interactions and small energy scales of the order of $\mathrm{meV}$, offer a highly tunable playground for different kinds of

*Corresponding author (email: yifeng@iphy.ac.cn) quantum criticality (see Figure 1) [3,4]. In $\mathrm{CeCu}_{2} \mathrm{Si}_{2}$, when the antiferromagnetism (AFM) and superconductivity are suppressed by the magnetic field or pressure, a NFL normal state is revealed with $\rho \sim T^{3 / 2}$ and $C / T \sim-\sqrt{T}$. These follow exactly the prediction of the conventional Hertz-MillisMoriya theory for itinerant magnetism, in which the AFM QCP occurs in a background of fully itinerant $f$ electrons as shown in Figure 1(b) and the low-energy physics is governed by the spin density wave (SDW) fluctuations near the AFM ordering wave vector [3]. By contrast, in $\mathrm{YbRh}_{2} \mathrm{Si}_{2}$, the AFM QCP seems to be accompanied by a Fermi surface reconstruction to incorporate the fully delocalized $f$ electrons following the Luttinger theorem. This underlies the so-called Kondo breakdown scenario for an unconventional QCP [3], which is thought to involve the simultaneous destruction of the AFM and the Kondo screening from opposite directions in the phase diagram of Figure 1(c). Later on, other proposals have also been put forward to explain the experiment. In the critical quasiparticle theory, the concept of Landau quasiparticles is extended to the critical region by assuming that the renormalization factor has a power law dependence on the quasiparticle energy [5]. Valence fluctuations may also play a role near the QCP [6]. In the two-fluid theory, the low-energy quantum dynamics is governed by both magnetic and hybridization fluctuations and the phase diagram is controlled by the interplay of the magnetic transition and the delocalization of the $f$ electrons [7], as illustrated in Figure 1. A consensus on the microscopic picture is yet to be reached. 

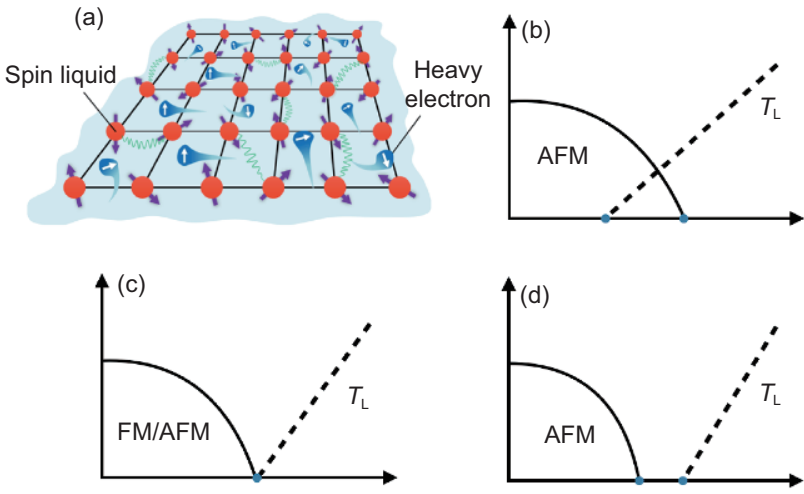

Figure 1 (Color online) (a) Illustration of the Kondo lattice with coexisting localized $f$ moments and itinerant heavy $f$ quasiparticles, whose instabilities cause various competing ground states, reprinted from ref. [4], with permission from IOP Publishing. Typical phase diagrams include: (b) the conventional one as in $\mathrm{CeCu}_{2} \mathrm{Si}_{2}$; (c) a single QCP with coincident magnetic and delocalization transitions as in $\mathrm{YbRh}_{2} \mathrm{Si}_{2}$ and $\mathrm{CeRh}_{6} \mathrm{Ge}_{4} ;(\mathrm{d})$ a quantum critical phase at zero temperature between separated magnetic and delocalization transitions as in CePdAl. In the two-fluid theory [4], the $f$ electrons turn gradually itinerant with lowering temperature. In all phase diagrams, the solid line denotes the AFM or FM transition of localized $f$ component and the dashed line marks the upper boundary $\left(T_{\mathrm{L}}\right)$ of full delocalization of all $f$ electrons. The low-energy quantum dynamics around the QCPs is governed by the interplay of their associated fluctuations.

Unlike those of AFM, no FM QCP has ever been observed in a stoichiometric compound until now. In the Hertz-MillisMoriya theory for itinerant ferromagnetism, the FM transition turns first order due to a non-analytic term in the lowenergy effective action of the order parameter. This has been confirmed in many compounds including $\mathrm{UTe}_{2}$, where spintriplet superconductivity has recently been found at ambient pressure [8]. Disorder could smooth the transition and introduce other complications. Thus, $\mathrm{CeRh}_{6} \mathrm{Ge}_{4}$ is a better counterexample than $\mathrm{YbNi}_{4}\left(\mathrm{P}_{0.92} \mathrm{As}_{0.08}\right)_{2}$. But one should not be too surprised, because neither of them is really an itinerant FM system. The $4 f$ electrons are at least partially localized in the FM state. Moreover, both compounds are quasi-onedimensional with highly anisotropic magnetic interactions and it is not clear if a FM QCP could occur at higher dimensionality. This, together with the possibility of finding the spin-triplet superconductivity near the FM QCP, poses a new challenge for future experimental investigations [9].

Yet another important advance is the discovery of a quantum critical phase in the frustrated Kondo lattice CePdAl reported last year in Nature Physics [10]. CePdAl has a distorted Kagome structure with an AFM ground state at am- bient conditions. Applying the magnetic field and pressure suppresses the AFM, but as illustrated in Figure 1(d), instead of a single QCP, there appears to be a finite parameter region at zero temperature showing nonmagnetic and NFL behavior with a $T$-linear resistivity. The nature of this intermediate phase is another important issue to be understood.

However, quantum criticality in heavy fermion materials is by no means limited to the above cases. It may involve other competing orders such as the quadrupole order or perhaps even more exotically, the "hidden" order as in $\mathrm{URu}_{2} \mathrm{Si}_{2}$. Their understanding is associated with the fundamental difficulty of strongly correlated physics, where the usual perturbative methods fail due to the lack of a small parameter. Exact numerical simulations are of limited use and new physical insights are desperately needed. The terminology "nonFermi liquid" is like calling a fruit "non-banana", an indefinite judgement in Kant's theory. The rich variety of anomalous phenomena urge for a new unified theory that is capable of categorizing all different kinds of heavy fermion quantum criticality. The discovery in $\mathrm{CeRh}_{6} \mathrm{Ge}_{4}$ is but another milestone along the road. The party is just beginning.

This work was supported by the National Key Research and Development Program of Ministry of Science and Technology of China (Grant No. 2017YFA0303103), and the National Natural Science Foundation of China (Grant Nos. 11774401, and 11974397).

1 B. Shen, Y. Zhang, Y. Komijani, M. Nicklas, R. Borth, A. Wang, Y. Chen, Z. Nie, R. Li, X. Lu, H. Lee, M. Smidman, F. Steglich, P. Coleman, and H. Yuan, Nature 579, 51 (2020), arXiv: 1907.10470.

2 A. Steppke, R. Kuchler, S. Lausberg, E. Lengyel, L. Steinke, R. Borth, T. Luhmann, C. Krellner, M. Nicklas, C. Geibel, F. Steglich, and M. Brando, Science 339, 933 (2013).

3 O. Stockert, and F. Steglich, Annu. Rev. Condens. Matter Phys. 2, 79 (2011); and references therein.

4 Y.-F. Yang, Rep. Prog. Phys. 79, 074501 (2016), arXiv: 1601.05894.

5 E. Abrahams, and P. Wolfle, Proc. Natl. Acad. Sci. USA 109, 3238 (2012).

6 K. Miyake, and S. Watanabe, J. Phys. Soc. Jpn. 83, 061006 (2014), arXiv: 1405.2382.

7 Y.-F. Yang, D. Pines, and G. Lonzarich, Proc. Natl. Acad. Sci. USA 114, 6250 (2017), arXiv: 1702.08132.

8 S. Ran, C. Eckberg, Q. P. Ding, Y. Furukawa, T. Metz, S. R. Saha, I. L. Liu, M. Zic, H. Kim, J. Paglione, and N. P. Butch, Science 365, 684 (2019).

9 Q. Liu, B. Shen, M. Smidman, R. Li, Z. Y. Nie, X. Y. Xiao, Y. Chen, H. Lee, and H. Q. Yuan, Sci. China-Phys. Mech. Astron. 61, 77411 (2018), arXiv: 1804.05477.

10 H. Zhao, J. Zhang, M. Lyu, S. Bachus, Y. Tokiwa, P. Gegenwart, S. Zhang, J. Cheng, Y.-F. Yang, G. Chen, Y. Isikawa, Q. Si, F. Steglich, and P. Sun, Nat. Phys. 15, 1261 (2019), arXiv: 1907.04255. 